BMC

Plant Biology

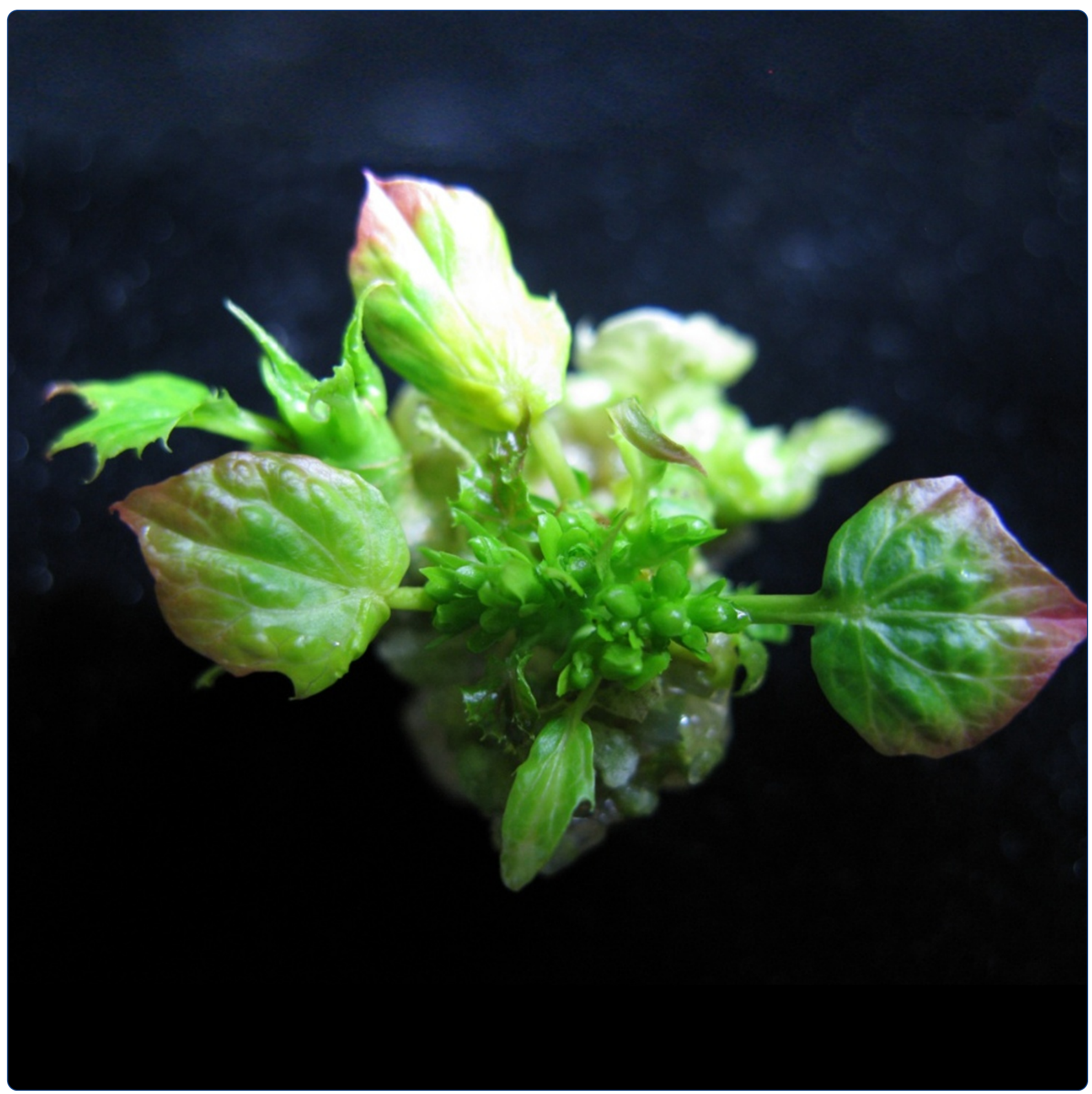

Isolation and functional characterization of JCFT, a FLOWERING LOCUS T (FT) homologous gene from the biofuel plant Jatropha curcas

Li et al. 


\title{
Isolation and functional characterization of JCFT, a FLOWERING LOCUS T (FT) homologous gene from the biofuel plant Jatropha curcas
}

\author{
Chaoqiong Li ${ }^{1,2}$, Li Luo ${ }^{3}$, Qiantang Fu', Longjian Niu ${ }^{1,4}$ and Zeng-Fu Xu ${ }^{1 *}$
}

\begin{abstract}
Background: Physic nut (Jatropha curcas L.) is a potential feedstock for biofuel production because Jatropha oil is highly suitable for the production of the biodiesel and bio-jet fuels. However, Jatropha exhibits low seed yield as a result of unreliable and poor flowering. FLOWERING LOCUS T(FT) -like genes are important flowering regulators in higher plants. To date, the flowering genes in Jatropha have not yet been identified or characterized.

Results: To better understand the genetic control of flowering in Jatropha, an FT homolog was isolated from Jatropha and designated as JCFT. Sequence analysis and phylogenetic relationship of JCFT revealed a high sequence similarity with the FT genes of Litchi chinensis, Populus nigra and other perennial plants. JCFT was expressed in all tissues of adult plants except young leaves, with the highest expression level in female flowers. Overexpression of $J_{C} F T$ in Arabidopsis and Jatropha using the constitutive promoter cauliflower mosaic virus $35 \mathrm{~S}$ or the phloem-specific promoter Arabidopsis SUCROSE TRANSPORTER 2 promoter resulted in an extremely early flowering phenotype. Furthermore, several flowering genes downstream of JCFT were up-regulated in the JCFT-overexpression transgenic plant lines.
\end{abstract}

Conclusions: JCFT may encode a florigen that acts as a key regulator in flowering pathway. This study is the first to functionally characterize a flowering gene, namely, JcFT, in the biofuel plant Jatropha.

Keywords: Biofuel, Early flowering, Florigen, FLOWERING LOCUS T, Physic nut

\section{Background}

Physic nut (Jatropha curcas L.) is a perennial plant that belongs to the Euphorbiaceae family, and is monoecious with male and female flowers borne on the same plant within the same inflorescence [1]. The potential benefit of growing Jatropha as a cash crop for biofuel in tropical and sub-tropical countries is now widely recognized [2-4]. Jatropha has been propagated as a unique and potential biodiesel plant owing to its multipurpose value, high oil content, adaptability to marginal lands in a variety of agro-climatic conditions, non-competitiveness with food production, and high biomass productivity $[2,5]$. The oil content of Jatropha seeds and the kernels ranges from $30 \%$ to $50 \%$ and $45 \%$ to $60 \%$ by weight, respectively. Oil

\footnotetext{
* Correspondence: zfxu@xtbg.ac.cn

'Key Laboratory of Tropical Plant Resources and Sustainable Use, Xishuangbanna Tropical Botanical Garden, Chinese Academy of Sciences, Menglun, Yunnan 666303, China

Full list of author information is available at the end of the article
}

from Jatropha contains high levels of polyunsaturated fatty acids, and it is therefore suitable as a fuel oil $[6,7]$. However, the potential of Jatropha as a biofuel plant is limited by its low seed production. Despite the clear evidence of the abundant biomass generated by Jatropha, it is not indicative of high seed productivity [8]. There are too many vegetative shoots in Jatropha, which could develop into reproductive shoots under suitable conditions. It is therefore imperative to reduce undesired vegetative growth. In addition to these considerations, unreliable and poor flowering are important factors that contribute to low seed productivity in Jatropha [9]. The FLOWERING LOCUS T $(F T)$ gene plays a crucial role in the transition from vegetative growth to flowering, which is a potent factor integrating the flowering signals. In this context, the function of JcFT, an FT homolog in Jatropha, was analyzed to improve the understanding of the flowering mechanism in Jatropha, which will be critical for the genetic improvement of this species. 
The transition from vegetative to reproductive growth in plants is regulated by both environmental and endogenous cues [10]. The genetic network of flowering has been investigated primarily in the model plant Arabidopsis, and five major genetically pathways control flowering initiation: the photoperiod, vernalization, gibberellin, autonomous and age pathways [11]. Recent advances in transgenic plants and traditional grafting studies have revealed that FT protein acts as a mobile flowering signal, whose ability to induce flowering involves longdistance transport $[12,13]$. The findings of many studies have helped establish the role of FT as a floral pathway integrator that respond to both environmental and endogenous flowering signals [14].

In Arabidopsis, FT is expressed in leaf phloem, and the FT protein subsequently moves to the shoot apex, where it forms a complex with the basic domain/leucine zipper protein FD. This FT/FD heterodimer activates the downstream floral meristem identity gene APETALA1 (AP1) $[12,15,16]$. FT-like genes have been isolated from many plants, including tomato [17], pumpkin [18], rice [19], barley [20], grape [21], apple [22], and potato [23], and the function of most FT genes is conserved [24].

In this study, we cloned and characterized the Jatropha FT homolog, JcFT. We also analyzed the function of
JcFT in floral induction using transgenic Arabidopsis and Jatropha.

\section{Results}

Cloning and sequence analysis of JcFT

A combined reverse transcriptase-polymerase chain reaction (RT-PCR) and rapid-amplification of cDNA ends (RACE) strategy was used to isolate an FT-like cDNA from Jatropha. JcFT cDNA (GenBank accession no. KF113881) encoded a 176-amino acid protein with $89 \%, 83 \%, 80 \%$, and $78 \%$ sequences identity with Litchi chinensis LcFT [25], Citrus unshiu CiFT [26], rice Hd3a [27], and Arabidopsis FT [28], respectively. The molecular weight and isoelectric point of the deduced protein were $20.03 \mathrm{kDa}$ and 6.82, respectively.

The genomic sequence of $J c F T$ consisted of four exons, which resembles the genomic structure of other FT genes (Figure 1A). A multiple alignment was performed using the JcFT sequence and the sequences of FT homologs from other species (Figure 1B). The conserved key amino acid residue Tyr (Y) found in FT homologs was identified at position 85 of the JcFT protein (Figure 1B). JcFT also contained two highly similar sequences to Arabidopsis FT in the 14-AA stretch known as "segment B" and in the LYN triad in "segment C" [29] (Figure 1B).
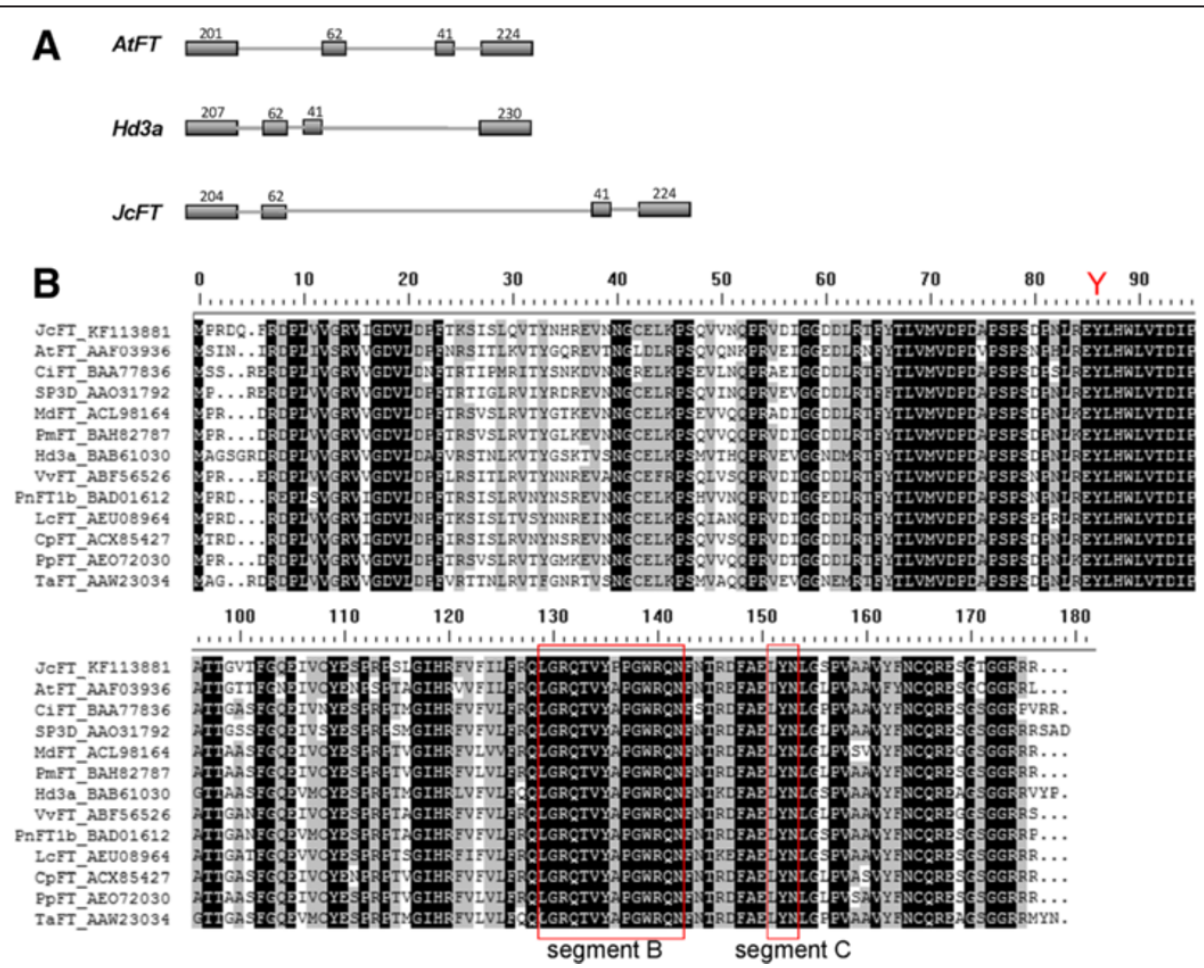

Figure 1 Comparison of JCFT and other FT-like genes. (A) Gene structures of JCFT, Hd3a, and AtFT. Boxes indicate exons and thin lines indicate introns. Exon sizes are indicated above each box. (B) Sequence alignment of amino acid sequences. Identical amino acid residues are shaded in black, and similar residues are shaded in gray. Dots denote gaps. Boxes indicating the 14-amino-acid stretch (segment B) and the LYN triad (segment $\mathrm{C}$ ), and "Y" indicating the highly conserved amino acid Tyr (Y). 
A phylogenetic tree was constructed to analyze the phylogenetic relationship between JcFT and the FTs from other angiosperms (Figure 2). The analysis revealed that the JcFT protein (indicated with a red-boxed) was more closely related to the FTs of perennial woody plants such as Litchi chinensis, instead of annual herbaceous plants such as Arabidopsis.

\section{Expression pattern of JCFT in Jatropha}

To assess the expression pattern of JcFT in Jatropha, we performed a quantitative RT-PCR (qRT-PCR) analysis using the specific primers listed in Table S1. JcFT was expressed in all adult plants tissues except young leaves (Figure 3). Interestingly, JcFT was primarily expressed in the reproductive organs rather than the leaves, where expression of a florigen-encoding gene is expressed (Figure 3).

\section{Constitutive overexpression and phloem-specific} expression of JCFT in Arabidopsis induces early flowering and complements the $\mathrm{ft}-10$ mutant phenotype

To determine whether $J c F T$ is involved in the regulation of flowering time, JcFT cDNA driven by the constitutive cauliflower mosaic virus 35S (CaMV 35S) promoter or the phloem-specific Arabidopsis SUCROSE TRANSPORTER
2 (SUC2) promoter was transformed into wild-type Arabidopsis Columbia (WT) and $f t-10$ mutant plants. An empty vector was transformed into WT as a control. Transgenic plants were confirmed by RT-PCR analysis of JcFT expression (Additional file 1: Figure S1A). Twentyfour and seven independent $\mathrm{T}_{0}$ transgenic lines were generated with the $35 \mathrm{~S}:: \mathrm{JCFT}$ construct in WT and $\mathrm{ft}$-10 mutant, respectively. For most of these lines, bolting occurred significantly earlier than in WT and $f t-10$ plants under inductive long-day (LD) conditions (Figures 4A and $5 \mathrm{~A})$.

We selected four independent homozygous lines in the $\mathrm{T}_{2}$ generation to examine the phenotypes. The L1 and L9 lines were created by transforming WT with the 35S:: $/ c F T$ construct, and the $\mathrm{C} 1$ and $\mathrm{C} 7$ lines harbored the construct in the $f t-10$ mutant background. Lines L1 and L9 bolted 8-14 days earlier and produced 6-11 fewer rosette leaves than the WT control under LD conditions, whereas no differences in bolting time were observed when comparing WT and the transgenic lines transformed with the empty vector (Figure 5A). Under non-inductive short-day (SD) conditions, all transgenic plants flowered much earlier than WT and the $f t-10$ mutant, both of which did not flower until 60 days after sowing in soil (Figures 4B and 5B). JcFT overexpression in Arabidopsis did not cause any

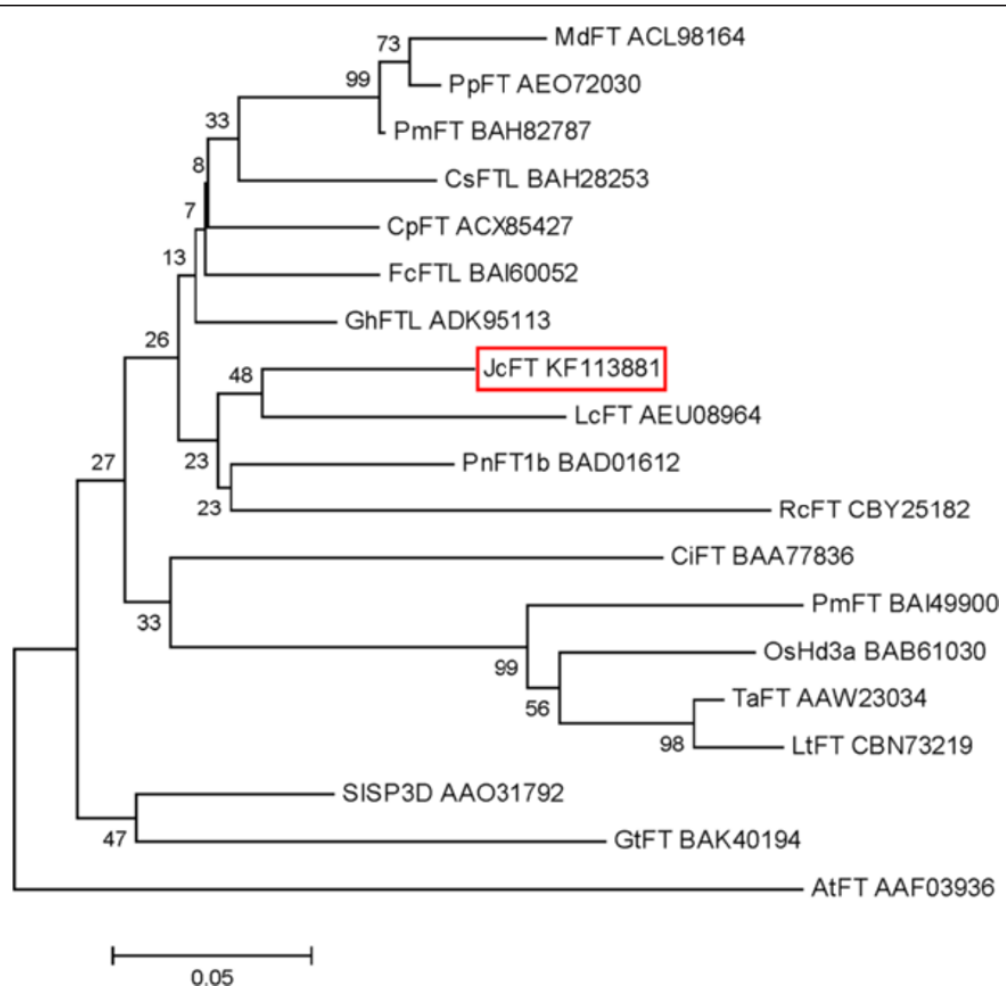

Figure 2 Phylogenetic analysis of the FT homologs from different plant species. Species abbreviations: At, Arabidopsis thaliana; Ci, Citrus unshiu; Cp, Carica papaya; Cs, Cucumis sativus; Fc, Ficus carica; Gh, Gossypium hirsutum; Gt, Gentiana triflora; Jc, Jatropha curcus; Lc, Litchi chinensis; Lt, Lolium temulentum; Md, Malus domestica; Os, Oryza sativa; Phm, Phyllostachys meyeri; Pm, Prunus mume; Pn, Populus nigra; Pp, Prunus persica; Rc, Rosa chinensis; Sl, Solanum lycopersicum; Ta, Triticum aestivum. 


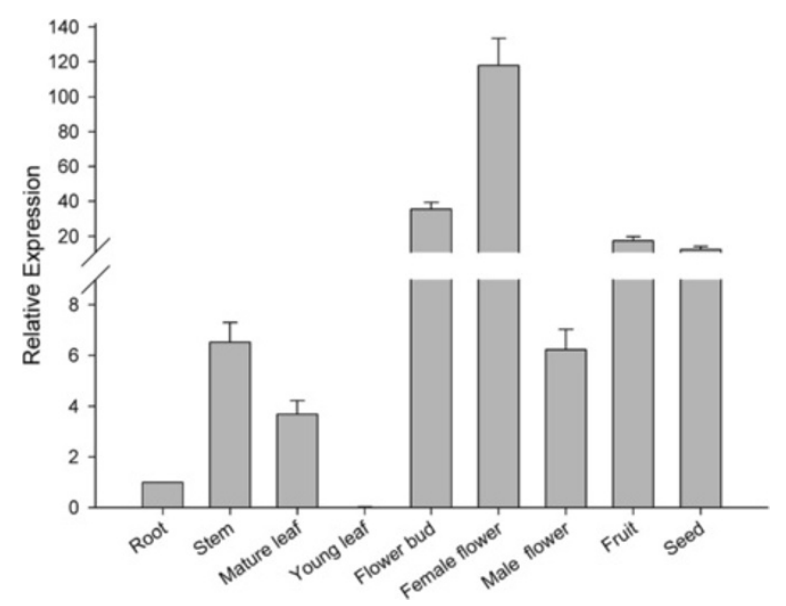

Figure 3 Expression of JCFT in various organs of three-year-old adult Jatropha. The QRT-PCR results were obtained from two independent biological replicates and three technical replicates for each sample. The levels of detected amplicons were normalized using the amplified products of the JcActin 1. The mRNA level in the root tissue was set as the standard with a value of 1 .

defects in flower development (Figure $4 \mathrm{C}$ and $4 \mathrm{D}$ ), but it did significantly reduce vegetative growth time. Further analysis indicated that the promotion of flowering in 35S:: JcFT transgenic Arabidopsis was correlated with a significant up-regulation of the flower meristem identity genes $A P 1$ and $L E A F Y(L F Y)$ (Additional file 2: Figure S1).

To determine whether $F T$-like genes are functionally conserved and active in vascular tissue, the phloemspecific promoter SUC2 has been used to drive the expression of FT-like genes in Arabidopsis and other species [12,30-32]. We obtained ten WT and eight $f t-10$ independent $\mathrm{T}_{0}$ transgenic lines harboring the SUC2:: IcFT construct. The S1 and S3 lines were created by transforming WT with the SUC2::ICFT construct, and the CS1 and CS4 lines harbored the construct in the $f t-10$ mutant background. Similar to the observations for the 35S:: JcFT transgenic lines, lines S1 and CS1 flowered much earlier than WT and $f t-10$, respectively. Lines S3 and CS4 flowered at approximately the same time and produced as many leaves as WT (24 days, 12 leaves) or flowered slightly earlier (Figure 5A) under LD conditions. Similar to the 35S:: $J C F T$ transgenic lines, all the SUC2:: $J_{c F T}$ transgenic lines flowered earlier than WT and $f t-10$ under SD conditions (Figure 5B).

Taken together, these findings demonstrated that ectopic expression of $J_{C F T}$ in Arabidopsis resulted in an early flowering phenotype.

\section{Overexpression of JcFT in Jatropha causes early flowering in vitro}

The transgenic analysis in Arabidopsis suggested that $J_{c} F T$ could be a floral activator in Jatropha. To test whether
$J_{c F T}$ similarly resulted in an early-flowering phenotype in Jatropha, we generated transgenic Jatropha with the 35S:: IcFT construct used for Arabidopsis transformation. Mature Jatropha cotyledons were used as explants for transformation, as previously described [33]. To our surprise, flower buds initiated directly from the Agrobacterium-transformed calli after in vitro culture for seven weeks (Figure 6A and 6B), whereas the control explants never produced flower buds under the same conditions. The in vitro cultured transgenic Jatropha also produced intact inflorescences, but the inflorescences did not produce as many small flowers as wild Jatropha in the field (Figure 6C and 6D). Nevertheless, these findings demonstrate that JcFT is a powerful inducer of flowering in Jatropha.

Although flower buds were produced in vitro, most were abortive and wilted several weeks later. A few flower buds developed into flowers (Figure 7A and 7C), but these flowers also wilted. Furthermore, these in vitro flowers were abnormal; for example, the petals of the female flower could not spread (Figure 7A). By removing the sepals and petals of female flower, the pistil was made visible (Figure 7B). Compared with the wild-type female flower (Figure 7F), the stigma of transgenic female flower was shorter (Figure 7B). An abnormal in vitro hermaphrodite flower of transgenic Jatropha (Figure 7C) had six stamens with very short filaments (Figure 7D) in contrast to the normal male flower (Figure 7E, G), which has ten stamens (Figure 7H). Consequently, no regenerated transgenic plants harboring $35 \mathrm{~S}:: . / c F T$ were obtained.

To determine whether JcFT overexpression in the transgenic in vitro flowering lines altered the expression of downstream flowering genes, such as SUPPRESSOR OF OVEREXPRESSION OF CONSTANS 1 (SOC1), LFY, and AP1 homologs in Jatropha [11], qRT-PCR analysis was performed with RNA extracted from apex of the 35S:: $J c F T$ transgenic and wild-type shoots cultured in vitro. As expected, the transcript levels of JcLFY, JcAP1, and JcAP3 were significantly up-regulated (Figure 8). JCSOC1 was also strongly up-regulated in the transgenic in vitro flowering lines (Figure 8), indicating that it is a target of $J_{C F T}$, which is consistent with the findings that SOC1 and AP1 are activated by the FT-FD complex in Arabidopsis [15,16,34].

\section{Discussion}

Chailakhyan [35] coined the term "florigen" to refer to the floral stimulus, but exactly what contributes florigen remains unclear. Evidence indicating that Arabidopsis FT protein acts as a long-distance signal to induce flowering was published half a decade ago [12]. Subsequent findings have led to the now widely accepted view that FT protein is the mobile flowering signal (florigen), or at the very least, a component of it [14]. In the present 

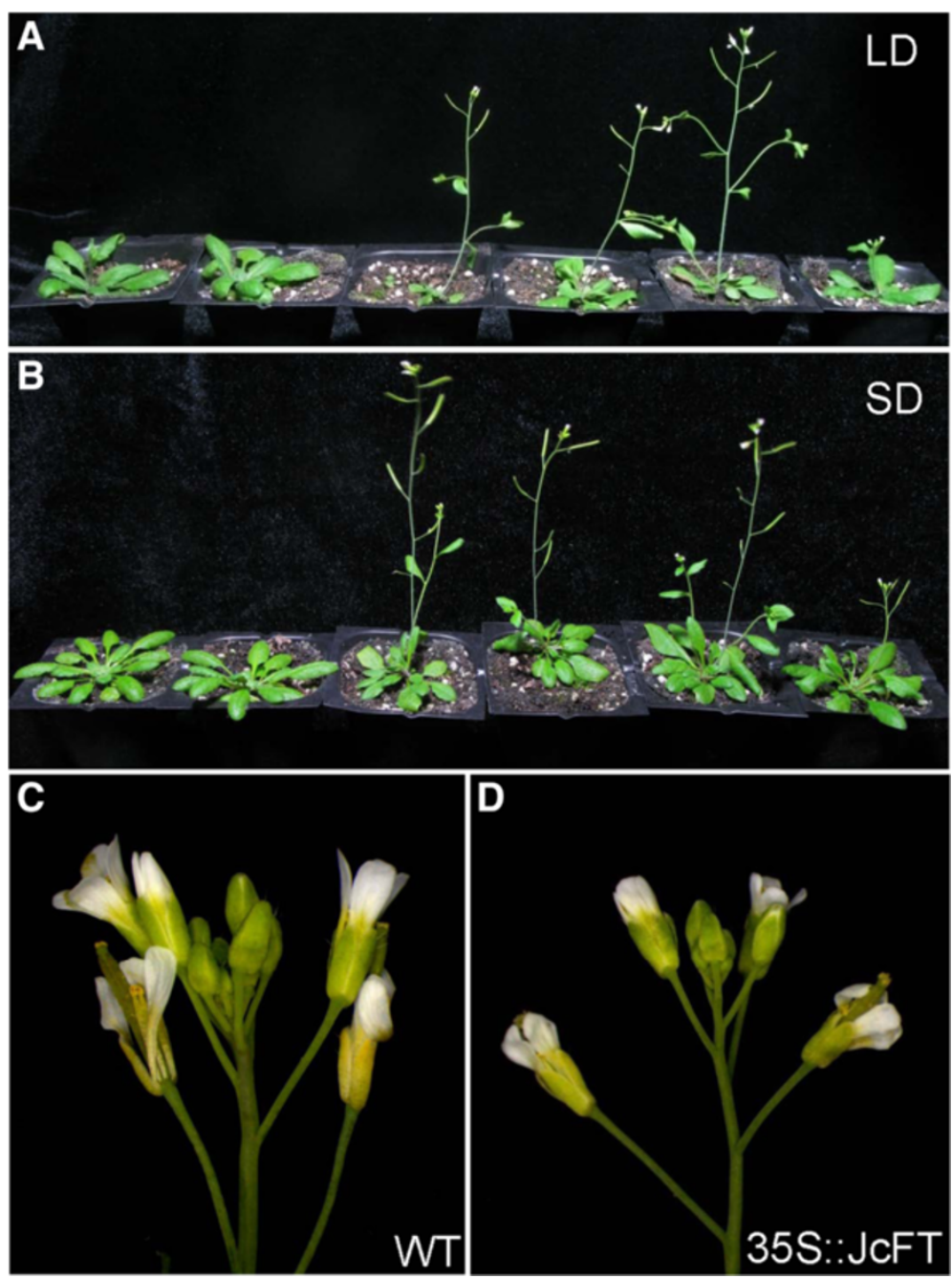

Figure 4 Ectopic expression of JcFT causes early flowering in transgenic Arabidopsis. Growth under LD conditions (A) and SD conditions (B) at 28 days and 45 days after germination, respectively. Left to right: WT, ft-10, 35S::JcFT in Col, SUC2::JcFT in Col, 35S::JCFT in ft-10, and SUC2::JCFT in ft-10. (C and D) Inflorescences of WT and 35S:.JCFT transgenic plants.

study, we found that JcFT encoded an FT homolog in Jatropha, and thus represented a potential flowering activator.

$F T$-like genes have been isolated from many plants. There are two members of the $F T$-like subclade in Arabidopsis [36], five in Lombardy poplar [37], ten in soybean [38], three in chrysanthemum [39], thirteen in rice, and fifteen in maize [40]. In Jatropha, we cloned only one member of the $F T$-like subclade, and only one $F T$-like gene was identified in the whole genome sequence data of Jatropha [41,42]. Many transgenic plants overexpressing $F T$ homologs exhibit an early flowering phenotype $[22,38,39,43,44]$, suggesting a conserved function of FT homologs in flowering induction in different plant species.

Although the leaf is generally expected to be the site where a florigen gene is translated into protein [13], many
FT-like genes are abundantly expressed in reproductive organs, such as flowers and immature siliques in Arabidopsis [28], flowers and pods in soybean [38], capsules in poplar [37], inflorescence axes in Curcuma kwangsiensis [32], and flowers and berries in grapevine [45]. In the present study, $J_{c} F T$ was mainly expressed in flowers, fruits, and seeds, with the highest expression level in female flowers, suggesting that $J_{C F T}$ may be involved in the development of reproductive organs. In fact, $F T$-like genes in various species play multifaceted roles in plant development in addition to the crucial role of FT homologs in flowering induction [10].

Transgenic Arabidopsis ectopically expressing the JcFT exhibited an early flowering phenotype compared with the control plants (Figures 4 and 5). Similarly, transgenic Jatropha overexpressing JcFT flowered in vitro during regeneration (Figure 6), which may have resulted from 

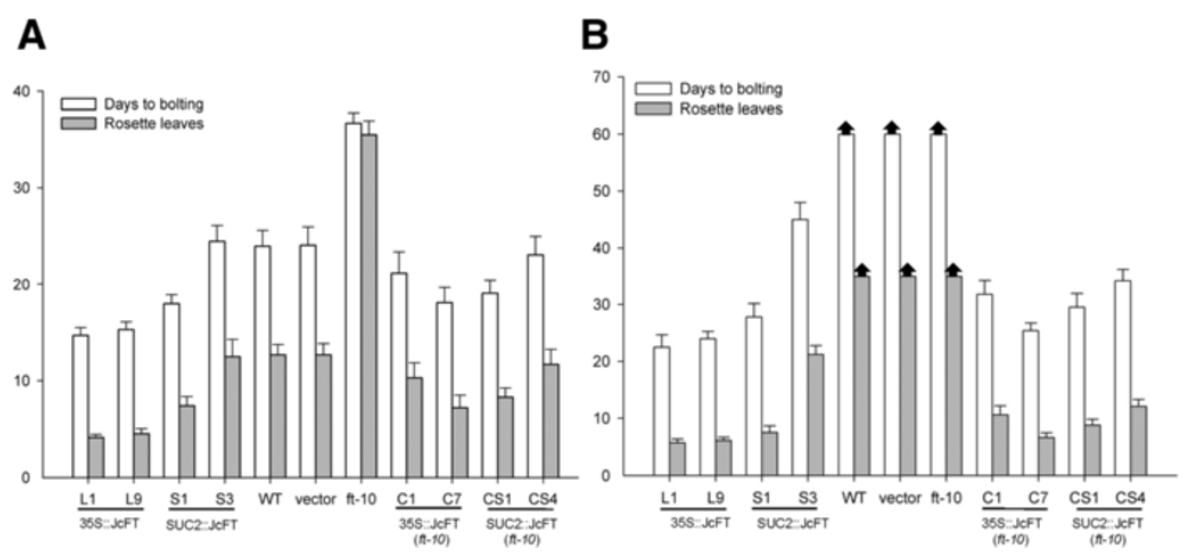

Figure 5 Ectopic expression of JCFT affects flowering in Arabidopsis. (A) Days and leaves to bolting for several JCFT overexpression (CaMV 35S) and phloem- specific expression (SUC2) transgenic Arabidopsis lines, empty vector-transformed plants, WT and mutant ft-10 plants grown under LD conditions. (B) Days and leaves to bolting for transgenic lines in the Col and ft-10 background grown under SD conditions. Values are means \pm SD of the results from ten plants of each transgenic line. Arrows at the top of bars for WT, empty vector-transformed Col and $\mathrm{ft}-10$ indicate that plants have not flowered.

the up-regulation of flowering gens downstream of $J_{c F T}$ (Figure 8). Unexpectedly, the transgenic Jatropha flower buds that were produced in vitro failed to develop normally into mature flowers. Many flower buds were abortive, and only a few developed into abnormal flowers. We supposed that the floral abnormalities and the failure of regeneration of the 35S::JcFT transgenic Jatropha plants could resulted from the ectopic overexpression of $J_{c} F T$ driven by the strong constitutive $35 \mathrm{~S}$ promoter. Consistent with this hypothesis, by using a phloem-specific promoter

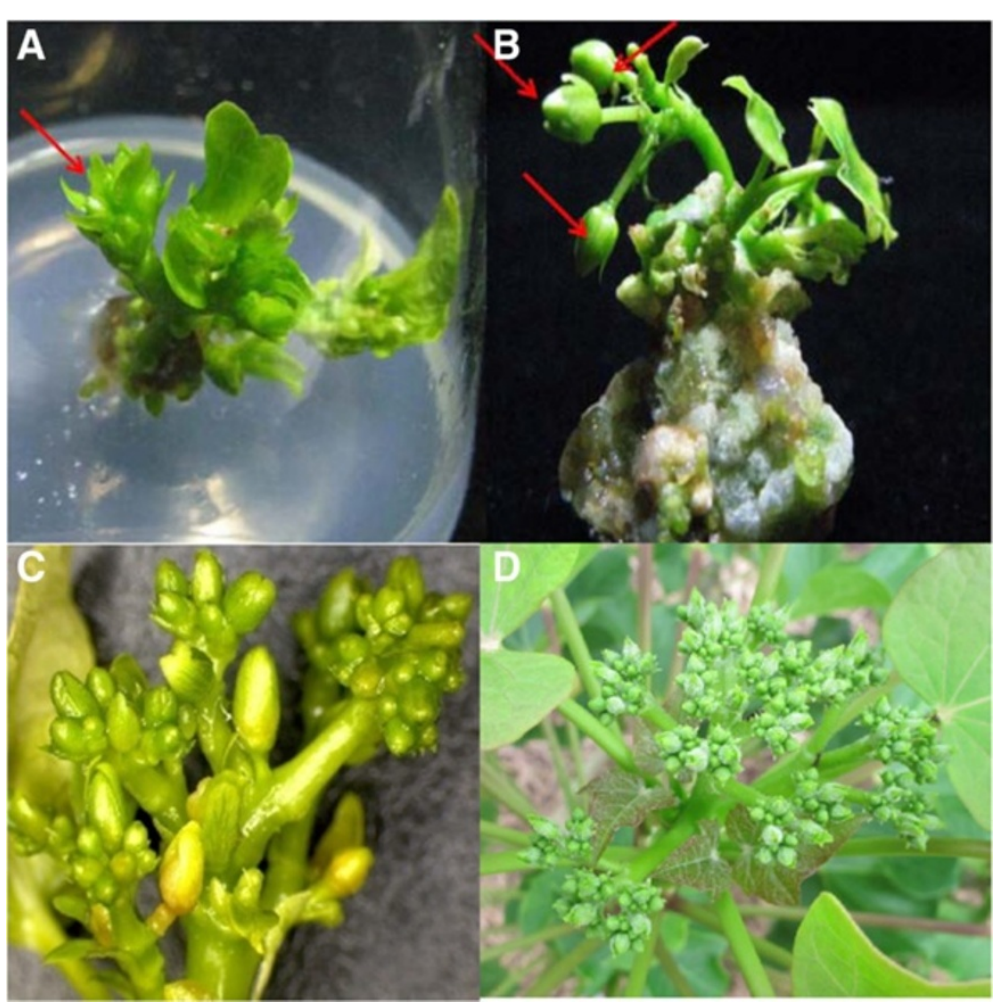

Figure 6 Early flowering of 35S::JcFT transgenic Jatropha cultured in vitro. (A and B) Flower buds of transgenic Jatropha cultured in vitro for seven weeks. (C) Inflorescence of transgenic Jatropha cultured in vitro. (D) Inflorescence of wild Jatropha in the field. Red arrows indicate flower buds. 

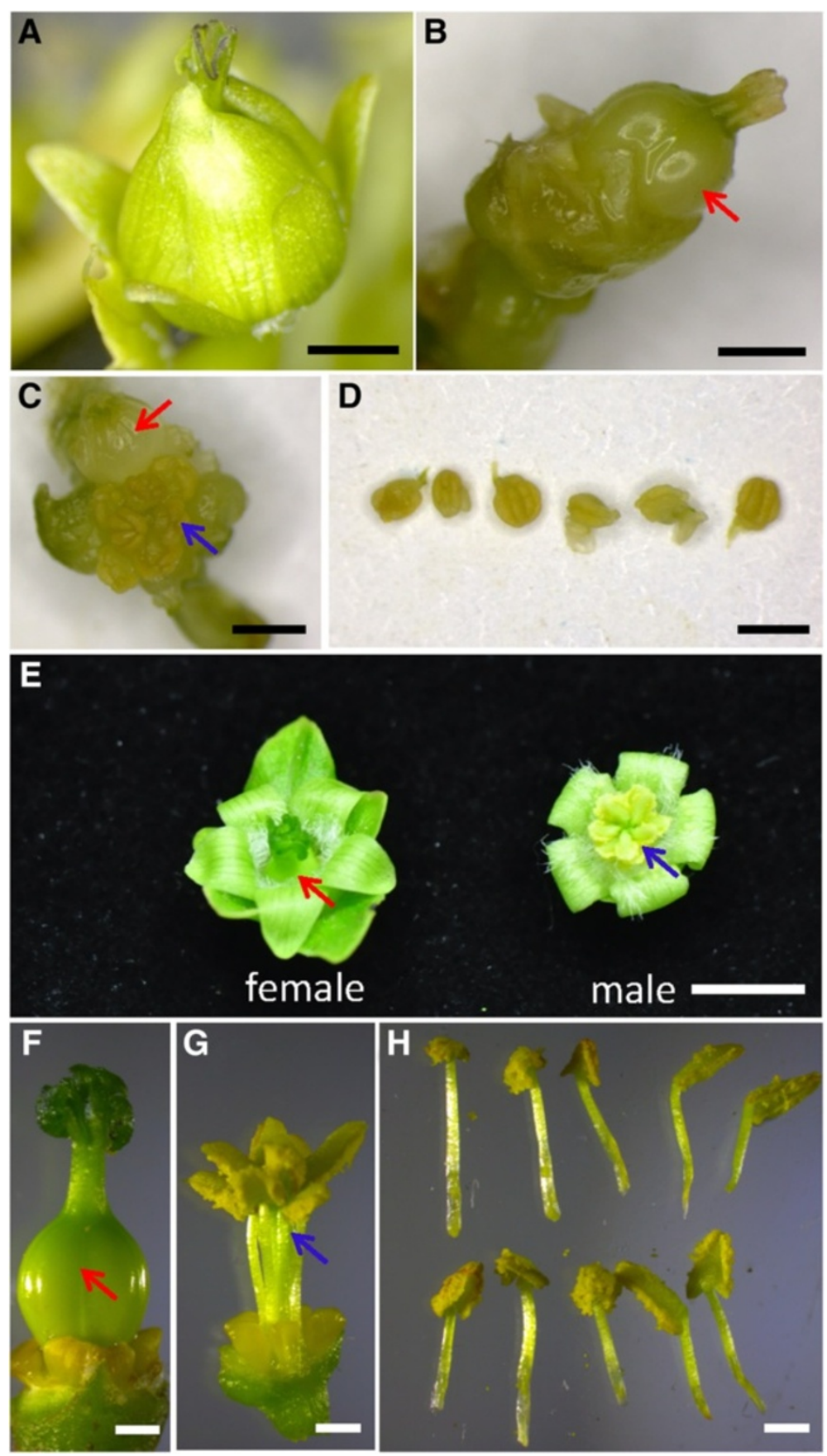

Figure 7 Abnormal flowers of transgenic Jatropha harboring 35S::JcFT. (A) A female flower of transgenic Jatropha cultured in vitro. (B) Pistil of a transgenic female flower. (C) An abnormal hermaphrodite flower of transgenic Jatropha cultured in vitro. (D) Abnormal stamens from an abnormal hermaphrodite flower of transgenic Jatropha shown in (C). (E) Normal female and male flowers of wild Jatropha grown in the field. (F) Pistil of a wild-type female flower. (G and $\mathbf{H})$ Stamens of a wild-type male flower. Bars in (A)-(D) and (F)-(H) represent $1 \mathrm{~mm}$, and bar in (E) represents $5 \mathrm{~mm}$. Red arrows indicate pistils, and blue arrows indicate stamens.

SUC2, we successfully obtained SUC2::JcFT transgenic Jatropha shoots, which were grafted onto rootstocks of wild-type Jatropha seedlings. The grafted SUC2:: IcFT transgenic Jatropha plants flowered earlier than did wildtype plants, and produced normal flowers (Additional file 2: Figure S2). Therefore, the production of normal transgenic Jatropha overexpressing JcFT for use in molecular breeding programs of Jatropha will likely require the use of weaker constitutive promoters [43], tissuespecific promoters [46], or inducible promoters [47] to 


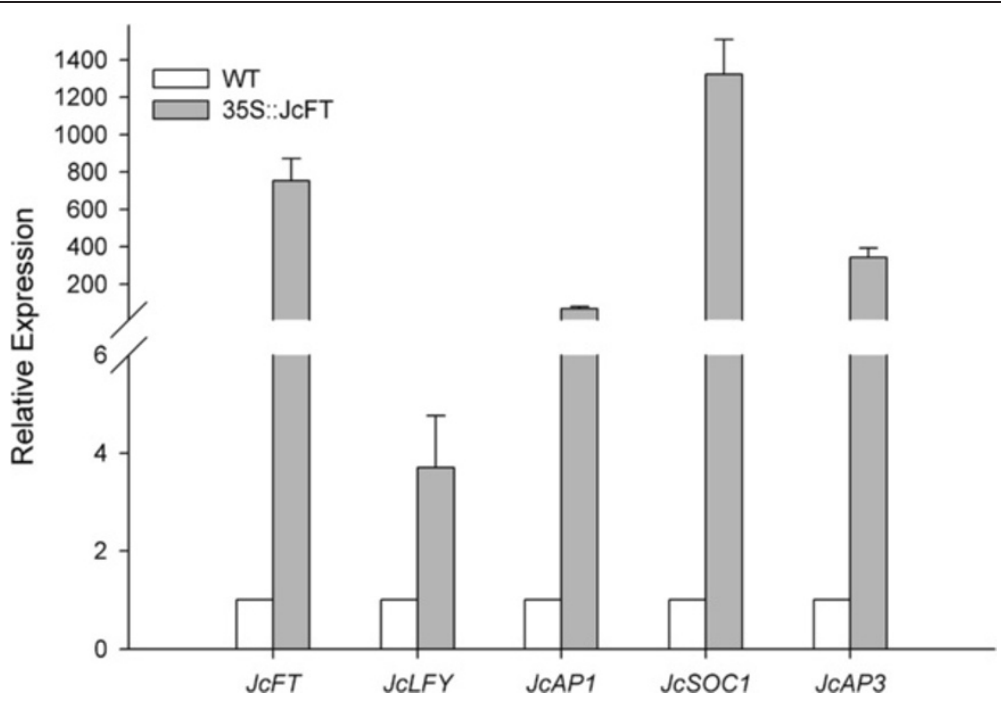

Figure 8 Quantitative RT-PCR analysis of flowering genes downstream of JcFT in WT and 35S::JcFT transgenic Jatropha. The qRT-PCR results were obtained using two independent biological replicates and three technical replicates for each RNA sample extracted from apex of the 35S.:.JCFT transgenic and wild-type (WT) shoots cultured in vitro. Transcript levels were normalized using JcActin 1 gene as a reference. The mRNA level in WT was set as the standard with a value of 1 .

confine the expression of the transgene $J_{c} F T$ to shoot meristems at an appropriate level. In addition, a loss of function analysis with a RNA interference construct targeted at $J_{c} F T$ will be necessary to determine the exact function of $J_{C} F T$ in Jatropha flowering.

\section{Conclusions}

The FT homolog of the biofuel plant Jatropha was isolated and characterized in the present study. JcFT is mainly expressed in the reproductive organs, including female flowers, fruits, and seeds. JcFT also induced early flowering in transgenic Arabidopsis and Jatropha, indicating that JcFT acts as a flowering promoter in Jatropha.

\section{Materials and methods}

\section{Plant materials and growth conditions}

The roots, stems, young leaves, mature leaves, flower buds, flowers, and fruits of Jatropha curcas L. were collected during the summer from the Xishuangbanna Tropical Botanical Garden of the Chinese Academy of Sciences, Mengla County, Yunnan Province in southwestern, China. Mature seeds were collected in autumn. All tissues prepared for qRT-PCR were immediately frozen in liquid $\mathrm{N}_{2}$ and stored at $-80^{\circ} \mathrm{C}$ until use.

WT Arabidopsis thaliana ecotype Columbia (Col-0), the $f t-10$ mutant (a gift from Dr. Tao Huang, Xiamen University), and the transgenic lines were grown in peat soil in plant growth chambers at $22 \pm 2^{\circ} \mathrm{C}$ under a $16 / 8 \mathrm{~h}$ (light/dark) or $8 / 16 \mathrm{~h}$ (light/dark) photoperiod, with coolwhite fluorescent lamps used for lighting. Transgenic plants in the $T_{2}$ homozygous generation were selected to examine flowering time and other phenotypes. For each genotype, ten plants were used to for characterization: the number of leaves was counted along with the number of days between sowing and when the first flower bud was visible.

\section{Cloning of JCFT CDNA}

Total RNA was extracted from the leaves of flowering Jatropha using the protocol described by Ding et al. [48] First-strand cDNA was synthesized using M-MLV-reverse transcriptase from TAKARA (Dalian, China) according to the manufacturer's instructions. To clone the conserved region of $J_{c} F T$ cDNA, a pair of primers, ZF632 and ZF633, was designed according to the conserved regions of $F T$ homologs from other plant species using the Primer Premier 5 software. The PCR products were isolated, cloned into the pMD19-T simple vector (TAKARA, Dalian, China), and sequenced. The cloned sequence was used to design gene-specific primers (GSPs) to amplify the cDNA $5^{\prime}$ and $3^{\prime}$ end. The primers were listed in Table S1. First round PCR and nested amplification were performed according to the instructions provided in the SMART $^{\mathrm{TM}}$ RACE CDNA Amplification Kit User Manual (Clontech). The PCR products were subsequently cloned into pMD19-T and sequenced.

The full length $J c F T$ cDNA was obtained by PCR using the primers JcFT-F and JcFT-R, which introduced KpnI and SalI recognition sites, respectively, to facilitate the transformation of JcFT into Arabidopsis and Jatropha. The PCR products were subsequently cloned into the pMD19-T and sequenced. 


\section{Sequence and phylogenetic analyses}

Sequence chromatograms were examined and edited using Chromas Version 2.23. Related sequences were identified using BLAST (http://www.ncbi.nlm.nih.gov/BLAST/). To determine the amino acid identities, sequences from the alignment were pairwise compared using DNAMAN 6.0. A phylogenetic tree based on the protein sequences was constructed using MEGA5.0 (http://www.megasoftware. net). The amino acid sequences of the PEBP family were assembled using ClustalX. A Neighbor-Joining phylogenetic tree was generated with MEGA 5.0 using the Poisson model with gamma-distributed rates and 10000 bootstrap replicates. The molecular weight and isoelectric point of the protein were analyzed on-line using ExPASy (http:// web.expasy.org/compute_pi/).

\section{Plant expression vector construction and Arabidopsis and Jatropha transformation}

To construct the plant overexpression vector $35 \mathrm{~S}:: / c F T$, the $J_{c F T}$ sequence was excised from the pMD19-T simple vector using the restriction enzymes $K p n I$ and SalI and then cloned into the pOCA30 vector containing the CaMV 35 S promoter and the $35 \mathrm{~S}$ enhancer. The SUC2 promoter was obtained by PCR from Arabidopsis genomic DNA using primers SUC2-F and SUC2-R, which introduced HindIII and KpnI recognition sites, respectively. The PCR products were cloned into pMD19-T and sequenced. To construct the SUC2:: $/ c F T$ plasmid, the $35 \mathrm{~S}$ promoter of the vector containing 35S:: $/ c F T$ was placed with the SUC2 promoter using the restriction enzymes HindIII and KpnI. The fidelity of the construct was confirmed by PCR and restriction digestion.

Transformation of WT Col-0 and ft-10 mutant plants with Agrobacterium strain EHA105 carrying the recombinant constructs was performed using the floral dip method [49]. Transgenic seedlings were selected for kanamycin resistance and confirmed by genomic PCR and RT-PCR.

Transformation of Jatropha with Agrobacterium strain LBA4404 carrying the overexpression construct was performed according to the protocol described by Pan et al. [33].

\section{Expression analysis by qRT-PCR}

Jatropha total RNA was extracted from frozen tissue as described by Ding et al. [48] Arabidopsis total RNA was extracted from frozen tissue using TRIzol reagent (Transgene, China). First-strand cDNA was synthesized using the PrimeScript ${ }^{\circ}$ RT Reagent Kit with gDNA Eraser (TAKARA, Dalian, China) according to the manufacturer's instructions. qRT-PCR was performed using $\mathrm{SYBR}^{\circ}$ Premix Ex $\operatorname{Taq}^{\text {Ti }}$ II (TAKARA) on a Roche 480 Real-Time PCR Detection System (Roche Diagnostics).
The primes used for qRT-PCR are listed in Table S1. qRT-PCR was performed using two independent biological replicates and three technical replicates for each sample. Data were analyzed using the $2^{-\Delta \Delta C T}$ method as described by Livak and Schmittgen [50]. The transcript levels of specific genes were normalized using Jatropha Actin1 or Arabidopsis Actin2.

\section{Availability of supporting data}

All the supporting data of this article are included as additional files (Additional file 1: Figure S1; Additional file 2: Figure S2; Additional file 3: Table S1).

\section{Additional files}

Additional file 1: Figure S1. Semi-quantitative (A) and quantitative (B) RT-PCR analysis of flowering genes downstream of FT in WT and transgenic Arabidopsis. Arabidopsis seedlings were collected 20 days after germination. For semi-quantitative RT-PCR, 25 cycles were used for the reference gene AtActin2, and 30 cycles were used for the target genes. The qRT-PCR results were obtained from three technical replicates for each sample. Values were normalized using AtActin2 gene as a reference. The mRNA level in WT was set as the standard with a value of 1.

Additional file 2: Figure S2. Early flowering of SUC2::JCFT transgenic Jatropha. Transgenic shoot grafted onto a non-transgenic rootstock showing the precocious flowers (red oval) forty days after grafting. Red arrows indicate the graft sites.

Additional file 3: Table S1. Primers used in this study.

\section{Abbreviations}

AP1: APETALA1; FT: FLOWERING LOCUS T; LFY: LEAFY; LD: long day; SD: short day; SOC1: SUPPRESSOR OF OVEREXPRESSION OF CONSTANS 1; SUC2: sucrose transporter 2; qRT-PCR: quantitative reverse transcriptase-polymerase chain reaction.

\section{Competing interests}

The authors declare that they have no competing interests.

\section{Authors' contributions}

$\mathrm{CL}$ and ZFX conceived the experiment and drafted the manuscript. LL cloned JCFT CDNA. CL constructed the vector and performed JCFT expression pattern analysis, Arabidopsis and Jatropha transformation, the transgenic plants bioassays. QF contributed to the data processing. LN collected the various Jatropha tissue samples. All authors read and approved the final manuscript.

\section{Authors' information}

$\mathrm{CL}$ and $\mathrm{LN}$ are PhD students, LL was a master student at the time of study, and QF is an associate professor, and ZFX is a professor and head of the laboratory.

\section{Acknowledgements}

We acknowledge Dr. Tao Huang for the Arabidopsis mutant $\mathrm{ft}-10$. This work was supported by funding from the Top Science and Technology Talents Scheme of Yunnan Province (2009C1123), the Natural Science Foundation of Yunnan Province (2011FA034) and the CAS 135 Program (XTBG-T02) awarded to Z.-F. Xu. The authors gratefully acknowledge the Central Laboratory of the Xishuangbanna Tropical Botanical Garden for providing the research facilities.

\section{Author details}

${ }^{1}$ Key Laboratory of Tropical Plant Resources and Sustainable Use, Xishuangbanna Tropical Botanical Garden, Chinese Academy of Sciences, Menglun, Yunnan 666303, China. ${ }^{2}$ University of Chinese Academy of Sciences, Beijing 100049, China. ${ }^{3}$ Key Laboratory of Gene Engineering of the 
Ministry of Education, and State Key Laboratory for Biocontrol, School of Life Sciences, Sun Yat-sen University, Guangzhou, Guangdong 510275, China. ${ }^{4}$ School of Life Sciences, University of Science and Technology of China, Hefei, Anhui 230027, China.

Received: 13 December 2013 Accepted: 2 May 2014 Published: 8 May 2014

\section{References}

1. Divakara B, Upadhyaya H, Wani S, Gowda C: Biology and genetic improvement of Jatropha curcas L.: A review. Appl Energy 2010, 87:732-742.

2. Akashi K: Jatropha research: A new frontier for biofuel development. Plant Biotechnol 2012, 29:121.

3. Pua F-I, Fang Z, Zakaria S, Guo F, Chia C-h: Direct production of biodiesel from high-acid value Jatropha oil with solid acid catalyst derived from lignin. Biotechnol Biofuels 2011, 4:1-8.

4. Khalil H, Aprilia N, Bhat A, Jawaid M, Paridah M, Rudi D: A Jatropha biomass as renewable materials for biocomposites and its applications. Renew Sust Energ Rev 2013, 22:667-685.

5. Pandey VC, Singh K, Singh JS, Kumar A, Singh B, Singh RP: Jatropha curcas: A potential biofuel plant for sustainable environmental development. Renew Sust Energy Rev 2012, 16:2870-2883.

6. Ong H, Mahlia T, Masjuki H, Norhasyima R: Comparison of palm oil, Jatropha curcas and Calophyllum inophyllum for biodiesel: a review. Renew Sust Energy Rev 2011, 15:3501-3515.

7. Pramanik K: Properties and use of Jatropha curcas oil and diesel fuel blends in compression ignition engine. Renew Energ 2003, 28:239-248.

8. Ghosh A, Chaudhary D, Reddy M, Rao S, Chikara J, Pandya J, Patolia J, Gandhi M, Adimurthy S, Vaghela N, Mishra S, Rathod MR, Prakash AR, Shethia BD, Upadhyay SC, Balakrishna V, Prakash CR, Ghosh PK: Prospects for Jatropha methyl ester (biodiesel) in India. Int J Enviro Stud 2007, 64:659-674.

9. Ghosh A, Chikara J, Chaudhary D, Prakash AR, Boricha G, Zala A: Paclobutrazol arrests vegetative growth and unveils unexpressed yield potential of Jatropha curcas. J Plant Growth Regul 2010, 29:307-315.

10. Pin P, Nilsson O: The multifaceted roles of FLOWERING LOCUS T in plant development. Plant Cell Environ 2012, 35:1742-1755.

11. Srikanth A, Schmid M: Regulation of flowering time: all roads lead to Rome. Cell Mol Life Sci 2011, 68:2013-2037.

12. Corbesier L, Vincent C, Jang S, Fornara F, Fan Q, Searle I, Giakountis A Farrona S, Gissot L, Turnbull C, Coupland G: FT protein movement contributes to long-distance signaling in floral induction of Arabidopsis. Science 2007, 316:1030-1033.

13. Turck F, Fornara F, Coupland G: Regulation and identity of florigen: FLOWERING LOCUS T moves center stage. Annu Rev Plant Biol 2008, 59:573-594

14. Wigge PA: FT, a mobile developmental signal in plants. Curr Biol 2011, 21:R374-R378

15. Abe M, Kobayashi Y, Yamamoto S, Daimon Y, Yamaguchi A, lkeda Y, Ichinok H, Notaguchi M, Goto K, Araki T: FD, a bZIP protein mediating signals from the floral pathway integrator FT at the shoot apex. Science 2005, 309:1052.

16. Wigge PA, Kim MC, Jaeger KE, Busch W, Schmid M, Lohmann JU, Weigel D: Integration of spatial and temporal information during floral induction in Arabidopsis. Science 2005, 309:1056.

17. Lifschitz E, Eviatar T, Rozman A, Shalit A, Goldshmidt A, Amsellem Z, Alvarez $J P$, Eshed $Y$ : The tomato FT ortholog triggers systemic signals that regulate growth and flowering and substitute for diverse environmental stimuli. Proc Natl Acad Sci 2006, 103:6398-6403.

18. Lin M-K, Belanger H, Lee Y-J, Varkonyi-Gasic E, Taoka K-I, Miura E, Xoconostle-Cázares B, Gendler K, Jorgensen RA, Phinney B, Lough TJ, Lucas WJ: FLOWERING LOCUS T protein may act as the long-distance florigenic signal in the cucurbits. Plant Cell 2007, 19:1488-1506.

19. Tamaki S, Matsuo S, Wong HL, Yokoi S, Shimamoto K: Hd3a protein is a mobile flowering signal in rice. Science 2007, 316:1033.

20. Faure S, Higgins J, Turner A, Laurie DA: The FLOWERING LOCUS T-like gene family in barley (Hordeum vulgare). Genetics 2007, 176:599-609.

21. Carmona MJ, Calonje M, Martínez-Zapater JM: The FT/TFL1 gene family in grapevine. Plant Mol Biol 2007, 63:637-650.
22. Kotoda N, Hayashi H, Suzuki M, Igarashi M, Hatsuyama Y, Kidou S-i, Igasaki T, Nishiguchi M, Yano K, Shimizu T, Takahashi S, Iwanami H, Moriya S, Abe K: Molecular characterization of FLOWERING LOCUS T-like genes of apple (Malusx domestica Borkh.). Plant Cell Physiol 2010, 51:561-575.

23. Navarro C, Abelenda JA, Cruz-Oró E, Cuéllar CA, Tamaki S, Silva J, Shimamoto K, Prat S: Control of flowering and storage organ formation in potato by FLOWERING LOCUS T. Nature 2011, 478:119-122.

24. Xu F, Rong $X$, Huang $X$, Cheng S: Recent advances of FLOWERING LOCUS T gene in higher plants. Int J Mol Sci 2012, 13:3773-3781.

25. Ding F, Peng H, He X, Li D, Zhu J, Qin X, Li H, Luo C, Cao H: Cloning and expression analysis of the FLOWERING LOCUS T (FT) homologous gene cDNA from Litchi chinensis. J Fruit Sci 2012, 29:75-80,160.

26. Hisada S, Akihama T, Endo T, Moriguchi T, Omura M: Expressed sequence tags of Citrus fruit during rapid cell development phase. J Am Society Hortic Sci 1997, 122:808-812.

27. Yamamoto T, Kuboki Y, Lin S, Sasaki T, Yano M: Fine mapping of quantitative trait loci $\mathrm{Hd}-1, \mathrm{Hd}-2$ and $\mathrm{Hd}-3$, controlling heading date of rice, as single Mendelian factors. Theor Appl Genet 1998, 97:37-44.

28. Kobayashi Y, Kaya H, Goto K, Iwabuchi M, Araki T: A pair of related genes with antagonistic roles in mediating flowering signals. Science 1999, 286:1960.

29. Ahn JH, Miller D, Winter VJ, Banfield MJ, Lee JH, Yoo SY, Henz SR, Brady RL, Weigel D: A divergent external loop confers antagonistic activity on floral regulators FT and TFL1. EMBO J 2006, 25:605-614.

30. Lazakis CM, Coneva V, Colasanti J: ZCN8 encodes a potential orthologue of Arabidopsis FT florigen that integrates both endogenous and photoperiod flowering signals in maize. J Exp Bot 2011, 62:4833-4842.

31. Flachowsky H, Peil A, Hanke M-V, Tränkner C, Szankowski I, Lehmann S: Functional characterization of two antagonistic acting flowering genes in apple MalusX domestica Borkh. In XXVIII International Horticultural Congress on Science and Horticulture for People (IHC2010): International Symposium on 929; 2010:351-356.

32. Varkonyi-Gasic E, Moss S, Voogd C, Wang T, Putterill J, Hellens RP: Homologs of $F T, C E N$ and FD respond to developmental and environmental signals affecting growth and flowering in the perennial vine kiwifruit. New Phytol 2013, 198:732-746.

33. Pan J, Fu Q, Xu Z-F: Agrobacterium tumefaciens-mediated transformation of biofuel plant Jatropha curcas using kanamycin selection. Afr Biotechnol 2010, 9:6477-6481.

34. Michaels SD: Flowering time regulation produces much fruit. Curr Opin Plant Biol 2009, 12:75-80

35. Chailakhyan MK: New facts in support of the hormonal theory of plant development. CR Acad Sci URSS 1936, 13:79-83.

36. Yamaguchi A, Kobayashi Y, Goto K, Abe M, Araki T: TWIN SISTER OF FT (TSF) acts as a floral pathway integrator redundantly with FT. Plant Cell Physiol 2005, 46:1175-1189

37. Igasaki T, Watanabe $Y$, Nishiguchi M, Kotoda N: The FLOWERING LOCUS T/ TERMINAL FLOWER 1 family in Lombardy poplar. Plant Cell Physiol 2008, 49:291-300

38. Sun H, Jia Z, Cao D, Jiang B, Wu C, Hou W, Liu Y, Fei Z, Zhao D, Han T: GmFT2a, a soybean homolog of FLOWERING LOCUS T, is involved in flowering transition and maintenance. PLoS One 2011, 6:e29238.

39. Oda A, Narumi T, Li T, Kando T, Higuchi Y, Sumitomo K, Fukai S, Hisamatsu T: CSFTL3, a chrysanthemum FLOWERING LOCUS T-like gene, is a key regulator of photoperiodic flowering in chrysanthemums. J Exp Bot 2012 63:1461-1477.

40. Danilevskaya ON, Meng X, Hou Z, Ananiev EV, Simmons CR: A genomic and expression compendium of the expanded PEBP gene family from maize. Plant Physiol 2008, 146:250-264.

41. Sato S, Hirakawa H, Isobe S, Fukai E, Watanabe A, Kato M, Kawashima K, Minami C, Muraki A, Nakazaki N, Takahashi C, Nakayama S, Kishida Y, Kohara M, Yamada M, Tsuruoka H, Sasamoto S, Tabata S, Aizu T, Toyoda A, Shin-i T, Minakuchi Y, Kohara Y, Fujiyama A, Tsuchimoto S, Kajiyama S, Makigano E, Ohmido N, Shibagaki N, Cartagena JA, et al: Sequence analysis of the genome of an oil-bearing tree, Jatropha curcas L. DNA Res 2011, 18:65-76.

42. Hirakawa $H$, Tsuchimoto S, Sakai H, Nakayama S, Fujishiro T, Kishida Y, Kohara M, Watanabe A, Yamada M, Aizu T, Toyoda A, Fujiyama A, Tabata S, Fukui K, Sato S: Upgraded genomic information of Jatropha curcas L. Plant Biotechnol 2012, 29:123-130.

43. Hsu C-Y, Liu Y, Luthe DS, Yuceer C: Poplar FT2 shortens the juvenile phase and promotes seasonal flowering. Plant Cell 2006, 18:1846-1861. 
44. Imamura T, Nakatsuka T, Higuchi A, Nishihara M, Takahashi H: The gentian orthologs of the FT/TFL1 gene family control floral initiation in Gentiana. Plant Cell Physiol 2011, 52:1031-1041.

45. Sreekantan L, Thomas MR: VVFT and VVMADS8, the grapevine homologues of the floral integrators $F T$ and $S O C 1$, have unique expression patterns in grapevine and hasten flowering in Arabidopsis. Funct Plant Biol 2006 33:1129-1139.

46. Tränkner $\mathrm{C}$, Lehmann $\mathrm{S}$, Hoenicka H, Hanke M-V, Fladung M, Lenhardt D, Dunemann F, Gau A, Schlangen K, Malnoy M, Flachowsky H: Over-expression of an $F T$-homologous gene of apple induces early flowering in annual and perennial plants. Planta 2010, 232:1309-1324.

47. Wenzel S, Flachowsky H, Hanke M-V: The Fast-track breeding approach can be improved by heat-induced expression of the FLOWERING LOCUS $T$ genes from poplar (Populus trichocarpa) in apple (Malus $\times$ domestica Borkh.). Plant Cell Tissue Organ Cult 2013, 115:127-137.

48. Ding $L-W$, Sun $Q-Y$, Wang Z-Y, Sun Y-B, Xu Z-F: Using silica particles to isolate total RNA from plant tissues recalcitrant to extraction in guanidine thiocyanate. Anal Biochem 2008, 374:426-428.

49. Clough SJ, Bent AF: Floral dip: a simplified method for Agrobacteriummediated transformation of Arabidopsis thaliana. Plant J 1998, 16:735-743.

50. Livak KJ, Schmittgen TD: Analysis of relative gene expression data using real-time quantitative PCR and the $2^{-\Delta \Delta C T}$ method. Methods 2001, 25:402-408.

doi:10.1186/1471-2229-14-125

Cite this article as: Li et al:: Isolation and functional characterization of JCFT, a FLOWERING LOCUS T (FT) homologous gene from the biofuel plant Jatropha curcas. BMC Plant Biology 2014 14:125.

\section{Submit your next manuscript to BioMed Central and take full advantage of:}

- Convenient online submission

- Thorough peer review

- No space constraints or color figure charges

- Immediate publication on acceptance

- Inclusion in PubMed, CAS, Scopus and Google Scholar

- Research which is freely available for redistribution 